\title{
Segmentation of Fingerprint Image Based on Gradient Magnitude and Coherence
}

\author{
Saparudin $^{1}$ and Ghazali Sulong ${ }^{2}$ \\ ${ }^{1}$ Faculty of Computer Science, Sriwijaya University, South Sumatera, Indonesia \\ ${ }^{2}$ Faculty of Computing, University Technology Malaysia, Johor Bahru, Malaysia \\ saparudin1204@yahoo.com¹, ghazali@spaceutm.edu.my²
}

\section{Article Info \\ Article history: \\ Received Jun 12, 2015 \\ Revised Aug 20, 2015 \\ Accepted Aug 26, 2015}

\section{Keyword:}

Fingerprint segmentation Gradient magnitude coherence Grey variance method

\begin{abstract}
Fingerprint image segmentation is an important pre-processing step in automatic fingerprint recognition system. A well-designed fingerprint segmentation technique can improve the accuracy in collecting clear fingerprint area and mark noise areas. The traditional grey variance segmentation method is widely and easily used, but it can hardly segment fingerprints with low contrast of high noise. To overcome the low image contrast, combining two-block feature; mean of gradient magnitude and coherence, where the fingerprint image is segmented into background, foreground or noisy regions, has been done. Except for the noisy regions in the foreground, there are still such noises existed in the background whose coherences are low, and are mistakenly assigned as foreground. A novel segmentation method based on combination local mean of grey-scale and local variance of gradient magnitude is presented in this paper. The proposed extraction begins with normalization of the fingerprint. Then, it is-followed by foreground region separation from the background. Finally, the gradient coherence approach is used to detect the noise regions existed in the foreground. Experimental results on NIST-Database14 fingerprint images indicate that the proposed method gives the impressive results.
\end{abstract}

Copyright (c) 2015 Institute of Advanced Engineering and Science. All rights reserved.

\section{Corresponding Author:}

Saparudin,

Departement of Informatic Engineering,

Universitas Sriwijaya,

Jalan Raya Palembang-Prabumulih, Km. 32, Indralaya, Ogan Ilir.

Email: saparudin1204@yahoo.com

\section{INTRODUCTION}

Fingerprint segmentation is a technique in which features sharing or regions with similar characteristics are identified and grouped collectively. In other words, the segmentation is splitting the fingerprint image into two regions, which are called foreground and background regions. The foreground regions correspond to clear fingerprint areas containing ridges and valleys, while background regions correspond to regions outside borders of fingerprint area, which do not contain any valid fingerprint information. If background regions have uniform grey-level and are lighter than foreground, then an approach based on local intensity could be effective for separating the foreground from the background, but in practice, fingerprint segmentation is sensitive to the quality of fingerprint image. Thus, the low quality of fingerprint image is a problem, which requires more robust segmentation techniques [11] [18].

The first problem is the presence of noise that resulted from dust and grease on the surface of livescan fingerprint scanners or ink-on-paper rolled fingerprint. The second problem is false tracings in the image acquisition. The third problem is the dryness or wetness that can influence the quality of ridges and valleys structure. The last one is the presence of an indistinct boundary when the features in windows of fixed size are used. Due to these problems, separating noise regions from the foreground regions are needed. Detection 
of noise regions in the foreground regions is a challenging problem in fingerprint segmentation, because the quality of the fingerprint area greatly contributes to improve the quality of ridges or valleys directions. Figure 1 shows a fingerprint image and its segmentation result that consists of background regions, foreground regions, and noise regions.

There are two general types of features used for fingerprint segmentation, i.e., block-wise and pixelwise features. Generally, pixel-wise feature of fingerprint segmentation provides accurate results, but its computational complexity and time consuming are markedly higher than most of block-wise features. Since pixel-wise based segmentation method is tedious and time consuming, block-wise features are more widely used in automatic fingerprint recognition systems [2] [18].

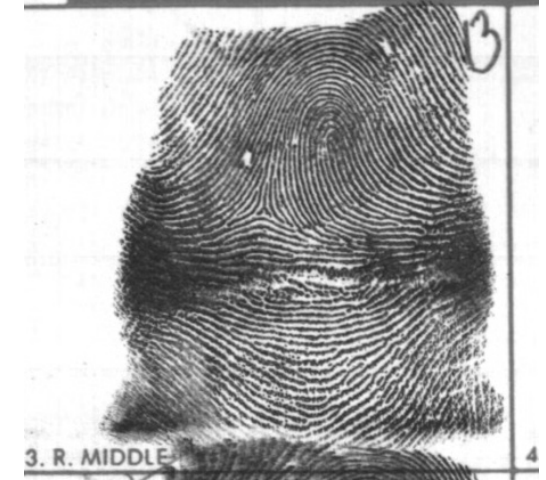

(a)

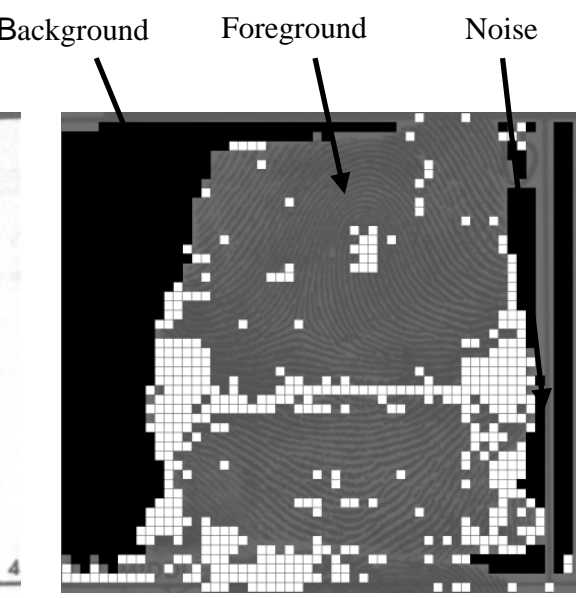

(b)

Figure 1. A sample of fingerprint segmentation results; (a) original fingerprint image, (b) background, foreground and noise patches of the segmented fingerprint image

\section{RELATED WORK}

Several features used in fingerprint segmentation are known from literatures, such as grey-scale statistical, local directionality, and consistency of orientation. Detailed explanations of these features are as follows.

\subsection{Grey-Scale Statistical Features}

Grey-scale statistical features in fingerprint segmentation include global mean, local mean, global variance, local variance, and histogram. In general, the mean of grey-level values in the foreground is lower than the values in the background. On the other hand, the variance of grey-level values in the foreground is higher than the background. However, mean and variance grey-scale-based algorithm does not work well on low quality fingerprint image [2] [9] [11].

Mehtre et al. computed directional image, representing local ridge orientation along eight different directions in blocks of size $16 \times 16$ pixels and segmented it using the block-wise histogram of the directional image values [12]. The histogram of the directional image technique gives a good result for low contrast and noisy images, but it fails for images with uniform regions. Contrarily, the variance grey-level method is not producing good results for low contrast image and instead it does produce good results for images with uniform regions. Also this method has no sense for clarity of ridges and their directions, and hence, cannot detect noisy regions as background. This leads Mehtre and Chatterjee uses the composite method by combining histogram and variance methods [13]. This method is reported to give good results for uniform regions, enhanced input images, and also poor for low contrast images.

Ratha et al. proposed a fingerprint segmentation to separate the fingerprint area to avoid extraction of feature in noisy and background areas using variance of grey-level in a direction orthogonal to the orientation field in each block of size $16 \times 16$. The angle of orientation field is quantized into 16 directions [15]. In [13], the variance at every pixel in a set of known directions used to decide whether the pixel is in the foreground. In addition, Ratha used the variance to decide the quality of the fingerprint image in terms of the image contrast of the block under consideration. The underlying assumption is that the noise regions have no 
directional dependence, whereas regions of interest exhibit have a very high variance in a direction orthogonal to the orientation of the pattern and a very low variance along the ridges. In other words, the background has low variance in all the directions. In [4] used the same methods as [15], but prior to segmentation process, fingerprint image is cropped using Candela’s approach [3] and is manually aligned in upright position.

Chen et al. proposed fingerprint segmentation using $12 \times 12$ pixel blocks and trained a linear classifier to separate foreground based on three features, namely: (i) the block cluster degree, (ii) the difference between local mean and global mean of grey-level, and (iii) the block variance of grey-level. This self-defined block cluster degree is a measure on how well the pixels of the block congregate within the block by comparing each pixel's intensity with the global mean intensity. A morphological operation is then applied during post-processing to normalize the results [5]. This method is claimed to provide satisfactory results for high quality image but has higher computational complexity than most unsupervised methods.

Feng et al. improved the grey-variance-based fingerprint segmentation algorithm by combining grey-level block mean and variance. They reported that this algorithm achieved more accurate and reliable segmented fingerprint results [6]. However, the robustness of their method yet to be tested on more complex fingerprints such as the ones that contain not only regular spikes but also foreign objects such as artefacts and handwritten annotations that normally found in raw fingerprints.

\subsection{Local Directionality Features}

Fingerprint image can be viewed as two distinct regions i.e., ridges' region and non-ridges’ region. The main purpose of the fingerprint segmentation is mainly to extract the ridges' region from the fingerprint image. With regards to that, some studies employed ridges orientation to segment the image. Generally, a ridge direction can be estimated by calculating its gradient, which normally performed in a pixel wise or block wise operations. The estimated gradient of a ridge is termed as orientation field.

Maio and Maltoni used mean of gradient magnitude of orientation field in image blocks to separate foreground from background. They observed that the gradient response is higher in the foreground compared to that in the background. By exploiting this information, they successfully extracted the foreground; however, they failed to identify the noise patches [10]. Zhang and Yan improved the above method by combining mean of gradient magnitude and coherence value [21]. They successfully segmented the fingerprint image into three parts namely, background, foreground and noisy regions; however, their assumptions that all noise areas are irrelevant and do not contain important information are proven wrong. Later, Qi and Xie proposed a more creative segmentation algorithm using the same mean magnitude of the gradient but this time they combined it with the variance of the gradient vector's directional image, instead [14]. This method is reported has achieved good results; however, noise patches in foreground area are failed to detect and are treated as part of the background.

Zhu et al. proposed a novel segmentation technique that gradually extract the foreground is based on correct orientation fields. The correctness of an orientation field is obtained by training the Neural Network (NN). The trained NN classifier is used to distinguish between the correct and incorrect orientation fields. Then, a foreground is gradually formed by adding on block by block of the corrected orientation fields, and its formation is exactly resemblance the famous region growing concept [22] [23]. The technique is evidently tedious and time consuming because every single orientation field has to be analysed and corrected should its bearing is off direction. Worse still, in real life application, fingerprints images may come in various qualities and in actual fact some are beyond our imagination.

Later, Yu et al. adopted a gradient projection method to exclude background region characterized by low grey-scale variation, and coarsely obtained the foreground region of the fingerprint image. In addition, noise regions, which contain smudges and stains, are excluded by using gradient coherence approach. Finally, morphological operations including edge detection are applied on the edges of the fingerprint image to obtain a smooth boundary of the foreground [20]. Although the published results evidently revealed that the extracted foregrounds are smooth but some parts of the foreground are unintentionally blackened. Hence, important information might be vanished that may lead to disappearance of singular points.

Finally, Teixeira and Leite proposed a rather complex fingerprint segmentation algorithm based on orientation fields, which exploits morphological mathematical transformations that include dilation and erosion [17]. Although their experiments yield promising results, the processes involved are rather tedious with high complexity. Worse still, the entire orientation fields are computed prior to the foreground extraction, which is considered against the normal practices, and is regarded as counter productive.

\subsection{Coherence Features}

A coherence feature represents the strength of local gradients centred at the target pixel, which has dominant representation. Generally, the coherence is also higher in the foreground, where the grey-level 
values are much smoother along the direction of the ridge. On the contrary, the coherence is comparatively lower at the region where there is a lot of spikes exist, which are emanated from noises such as stains and smudges [20]. It seems that the coherence is very promising to be used as a single feature to segment the foreground; however it is not sufficient for robusting segmentation. Therefore, a systematic combination of several features is necessary [2] [18].

Historically, the word coherence was first proposed by Kass and Witkin who define it as the norm of the sum of orientation vectors divided by the sum of their individual norms; this scalar always lies in the range of $[0,1]$. Orientations with parallel directions provide maximum coherence (i.e. its value is 1 ), whereas orientations with opposite directions give minimum coherence (i.e. its value is 0) [24].

Bazen and Gerez proposed a pixel-wise segmentation technique based on the coherence, while morphology is used to obtain smooth regions. The segmentation method is capable of successfully identifying very noisy region in the fingerprint [1]. Later in 2001, they improved the technique using three different features namely; coherence, local mean, and local variance, which are computed for each overlapped block of pixel [2]. The segmentation process is carried out on pixel-by-pixel basis in which the foreground separation is performed using a linear classifier. Then, a morphological operation is applied as post processing to obtain perfect clusters and to reduce categorization errors. Their experimental results showed that the method provides accurate results; however, its computational complexity is markedly higher than most of the described block-wise approaches. Moreover, Yin, et al. proposed a novel pixel-wise fingerprint segmentation approach based on quadric surface model [19]. They claimed that their proposed method has significantly reduced segmentation errors as opposed to that of the linear classifier.

Meanwhile, Klein et al. adopted four different pixel based features namely, grey-scale mean, greyscale variance, gradient coherence and Gabor response for the fingerprint segmentation. The segmented fingerprint image is decomposed into three parts viz. foreground, background and low-quality regions. In addition, a hidden Markov model (HMM) is applied to resolve the fragmented foregrounds instead of a common morphological operation. The pixel features are modelled as the output of a hidden Markov process [8]. The performance of HMM-based segmentation highly depends on the choice of pixel features. Their experimental results revealed that the outcomes are very encouraging with less fragmented foreground. In addition, the categorization of low-quality region provides an extra advantage that the information in this region is not totally discarded. However, the performance of the HMM is greatly relied on the choices of features used and number of state assigned for the background, foreground and low-quality regions. In actual fact, having too many features and states will increase the computational complexity and as well as computing time.

Zhang and Yan proposed the fingerprint segmentation using two-block features; mean of gradient magnitude and coherence. The fingerprint image is first convolved with a 2D Gaussian filter. The gradients in horizontal and vertical directions are estimated using Sobel operator. The mean of gradient magnitude is then compared with a threshold value of the gradient. They defined "invalid regions" in the foreground as the sets of connected elements with low coherence value. The fingerprint image is segmented into background, foreground or noisy regions [21]. Except for the noisy regions in the foreground, there are still such noises existed in the background whose coherences are low, and are mistakenly assigned as foreground.

\section{PROPOSED METHOD}

Foreground extraction is actually part of fingerprint image segmentation, which aims to separate foreground from its background and other foreign objects like artefacts and handwritten annotations, which are common in inked fingerprints. It also tasks for detecting noise regions found in the foreground.

The proposed extraction begins with normalization of the fingerprint's intensity values by adopting Hong's normalization approach [7]. Then, it is followed by foreground region separation from the background using the proposed segmentation technique. Finally, the gradient coherence approach, which is pioneered, by Zhang and Yan is adopted to detect the noise regions existed in the foreground. Diagrammatically, the method is displayed in Figure 2.

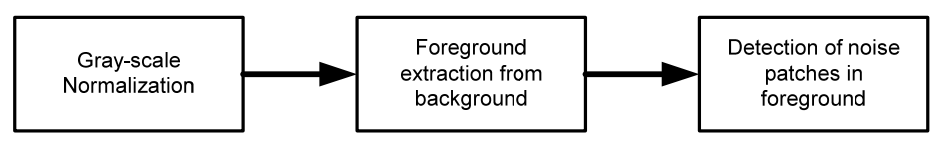

Figure 2. The fingerprint segmentation scheme 


\subsection{Grey-Scale Normalization}

Normally, the intensity value of fingerprint images is greatly varied from one print to another over time of capturing. As a result, there are prints whose intensity values concentrated in the upper-range of greylevels, for instance 128 - 255, which indicates bright images or over-exposure. On the contrary, there are fingerprints whose grey-levels ranging from $0-128$ or lower-range, which indicates dark images or underexposure. The uneven or irregular distribution of light intensities may affect the statistical information of the image such as mean and variance of grey-levels, and therefore normalization is needed. This normalization process aims at reducing variation in grey-level values along ridges and valleys without changing the clarity of their structures. Therefore, the input fingerprint image is standardized to a desired mean and variance. The Normalization method proposed in [7] consists of three steps: Firstly, global mean value of fingerprint image is determined. Secondly, global variance value of fingerprint image is computed. Finally, new intensity values are calculated.

Detailed process of the normalization is performed as follows:

1. Let $I(m, n)$ denote the grey-level or intensity value of the pixel at the $m$-th row and $n$-th column of $W \times H$ pixels of fingerprint image size. Let $M g$ and $V g$ denote the global mean and global variance values of fingerprint image, respectively.

2. Calculate the normalized grey-level value at pixel $(m, n)$ of fingerprint image, which is denoted by $N(m, n)$, and is defined as follows:

$$
N(m, n)=\left\{\begin{array}{lr}
\sum_{m=0}^{W-1 H-1} \sum_{n=0}^{H}\left(M g_{0}+\sqrt{\frac{V g_{0}(I(m, n)-M g)^{2}}{V g}}\right) & \text { if } I(m, n)>M g \\
\sum_{m=0}^{W-1} \sum_{n=0}^{H-1}\left(M g_{0}-\sqrt{\frac{V g_{0}(I(m, n)-M g)^{2}}{V g}}\right) & \text { otherwise }
\end{array}\right.
$$

where $M g_{0}$ and $V g_{0}$ are the desired mean and variance values, respectively. Ideally, the recommended value for both $M g_{0}$ and $V g_{0}$ is 100 .

\subsection{Proposed Foreground Extraction Method}

Once the normalised grey-level values of the fingerprint image are obtained, the next process is to extract the foreground from the fingerprint image. The process is done based on block-by-block basis starting from top left corner and ended at bottom right corner. With regard to that, a new segmentation approach, which combines local mean value of the normalised grey-level and local variance value of the gradient magnitude, is proposed. This method consists of three main steps: First, global mean (i.e. Mn in short) and local mean (i.e. $M b(i, j)$ in short) values of normalized fingerprint image are calculated. Second, local variance (i.e. denoted by $\operatorname{Vgr}(i, j))$ and threshold (i.e. $G_{t h}$ in short) values of gradient magnitude are computed. Finally, the target block is assigned as a part of foreground if the following conditions are fulfilled: (i) if the local mean is smaller than global mean, or (ii) the local variance is greater than the threshold. Diagrammatically, this process is displayed in Figure 3. 


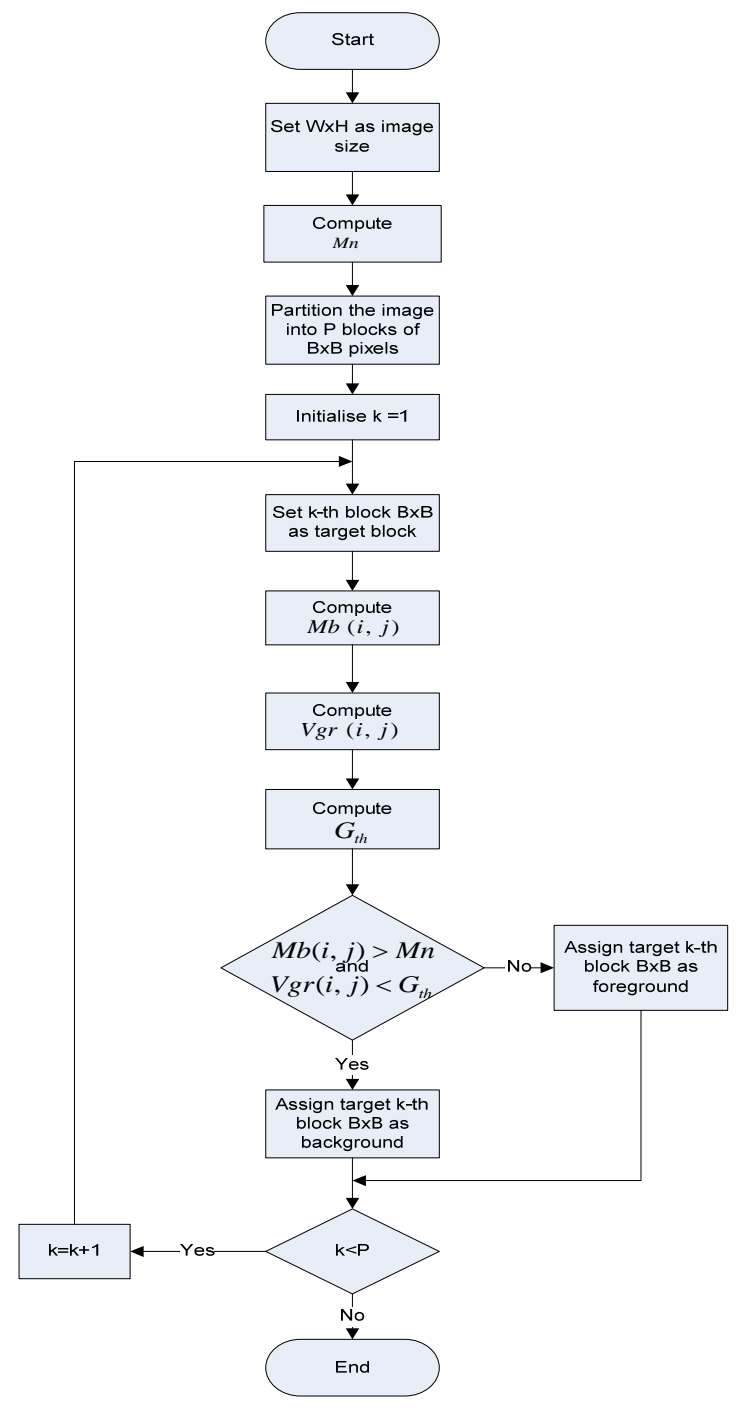

Figure 3. Flowchart of foreground extraction [16]

a. Computation of global mean and local mean values

Global mean is obtained by computing the average of grey-scale values of the whole normalized image, whereas local mean value is computed based on block of pixels. The calculation is performed as follows.

1. Let $W \times H$ be the size of the normalized image. Let $B \times B$ pixels be a non-overlapping block of the normalized image. In this case $B=16$. Let $N(u, v)$ be the intensity value of the pixel at the $u$-th row and $v$-th column of the $B \times B$ block. Let $P$ be the number of blocks in the entire image.

2. Calculate the global mean value, $M n$. Calculate the local mean of each block using following equation.

$$
M b(i, j)=\frac{\sum_{u=i}^{i+B-1} \sum_{v=j}^{j+B-1} N(u, v)}{B \times B},
$$

where $(i, j)$ is first pixel at $i$-th row and $j$-th column of the $B \times B$ block, $i=0,16,32, \ldots, W-16$, and $j=0,16,32, \ldots, H-16$.

b. Computation of local variance of gradient magnitude

The local variance of gradient magnitude of each block is computed according to the following steps:

IJECE Vol. 5, No. 5, October 2015 : 1202 - 1215 
1. For each pixel $(m, n)$ of the normalized fingerprint image $N(m, n)$; estimated gradients in horizontal and vertical directions, which are symbolized by $G_{x}(m, n)$ and $G_{y}(m, n)$, respectively are computed using the following Sobel mask $3 \times 3$ operators. Horizontal Sobel mask operator $S_{x}(p, q)$ and vertical Sobel mask operator $S_{y}(p, q)$

$$
\begin{aligned}
& G_{x}(m, n)=\sum_{p=-1}^{1} \sum_{q=-1}^{1}\left(S_{x}(p, q) \times N(m+p, n+q)\right) \\
& G_{y}(m, n)=\sum_{p=-1}^{1} \sum_{q=-1}^{1}\left(S_{y}(p, q) \times N(m+p, n+q)\right)
\end{aligned}
$$

2. Calculate the gradient magnitude $|G r(m, n)|$ for each pixel $(m, n)$ as follows.

$$
|G r(m, n)|=\sqrt{\left(G_{x}^{2}(m, n)+G_{y}^{2}(m, n)\right)}
$$

3. Determine the threshold value $G_{t h}$ of the gradient magnitudes using Zhang and Yan's method as follows: 3.1 Let $|\operatorname{Gr}(m, n)|$ denote the gradient magnitude at each pixel $(m, n)$ of the $W \times H$ image size.

3.2 Determine the maximum and the minimum of the gradient magnitudes, $|G r(m, n)|_{\max }$ and $|G r(m, n)|_{\min }$, respectively.

3.3 Calculate threshold value using the following equation.

$$
G_{t h}=c \times\left(|G r(m, n)|_{\max }-|G r(m, n)|_{\min }\right)+|G r(m, n)|_{\min }
$$

where $C$ is the threshold factor that can be chosen within a range of $[0.05,0.3]$ depending on image contrast. A smaller value of $C$ will encourage the block to become foreground, while larger value will transform the block to background. Empirically, $c=0.1$ is chosen.

4. Local variance of gradient magnitude can be determined as follows.

4.1 Let $|G r(u, v)|$ be the gradient magnitude of the pixel at $u$-th row and $v$-th column in the $B \times B$ block.

4.2 Calculate the local mean values of gradient magnitude $\operatorname{Mgr}(i, j)$ of each block $(i, j)$ as follows:

$$
\operatorname{Mgr}(i, j)=\frac{\sum_{u=i}^{i+B-1} \sum_{v=j}^{j+B-1}|G r(u, v)|}{B \times B}
$$

where $i=0,16,32, \ldots, W-16$, and $j=0,16,32, \ldots, H-16$.

4.3 Calculate the local variance values of gradient magnitude $\operatorname{Vgr}(i, j)$ of each block $(i, j)$ that are defined as follows:

$$
\operatorname{Vgr}(i, j)=\frac{\sum_{u=i}^{i+B-1} \sum_{v=j}^{j+B-1}(|G r(u, v)|-\operatorname{Mgr}(i, j))^{2}}{B \times B}
$$

where $i=0,16,32, \ldots, W-1$, and $j=0,16,32, \ldots, H-1$.

c. If ( $M b(i, j)>M n$ and $\left.\operatorname{Vgr}(i, j)<G_{t h}\right)$, then the target block is assigned as a part of background region, otherwise is designated as foreground region. 


\subsection{Noise Areas Identification and Marking Using Gradient Coherence}

Generally, gradient coherence is used to describe the variation of grey-level values in an image. It can also be applied to investigate on how does each pixels-block behaves in terms of its gradient value in relation to fingerprint ridge flows. The larger value indicates that every pixel of the block shares a common direction, which is in accordance to ridge direction. On the contrary, the smaller value signifies that majority of the pixels have non-uniform directions, and does not resemble true ridge flow. The gradient coherence value is usually larger in foreground of the fingerprint image, where the grey values are much smoother along the direction of the ridge than that at the perpendicular direction of the ridge. The gradient coherence measures range in $[0,1]$. Gradient coherence value of 0 indicates that the gradients in the block are equally distributed over all directions. On the other hand, gradient coherence value of 1 indicates all pixels of the block share the same orientation. Since gradient coherence is based on the block information of the fingerprint image, the fingerprint image is divided into non-overlapping blocks of $B \times B$ sized, in this case $B=16$. For a given normalized fingerprint image, gradient coherence $\operatorname{Coh}(i, j)$ of each block at pixel $(i, j)$ is calculated as follows:

1. Let $G_{x}(u, v)$ and $G_{y}(u, v)$ denote the gradients in $x$ and $y$ directions of the pixel at $u$-th row and $v$-th column in the $B \times B$ block.

2. Calculate the gradient coherence $\operatorname{Coh}(i, j)$ as follows

$$
\operatorname{Coh}(i, j)=\frac{\sqrt{V_{x}(i, j)^{2}+V_{y}(i, j)^{2}}}{V_{z}(i, j)},
$$

where

$$
\begin{aligned}
& V_{x}(i, j)=\sum_{u=i}^{i+B-1} \sum_{v=j}^{j+B-1}\left(G_{x}^{2}(u, v)-G_{y}^{2}(u, v)\right) \\
& V_{y}(i, j)=\sum_{u=i}^{i+B-1} \sum_{v=j}^{j+B-1} 2 G_{x}(u, v) G_{y}(u, v) \\
& V_{z}(i, j)=\sum_{u=i}^{i+B-1} \sum_{v=j}^{j+B-1}\left(G_{x}^{2}(u, v)+G_{y}^{2}(u, v)\right)
\end{aligned}
$$

where $i=0,16,32, \ldots, W-16$, and $j=0,16,32, \ldots, H-16$.

An example of the resultant image after undergone the above process is given in Figure 4. In this case, $\operatorname{Coh}(i, j)<=0.5$.

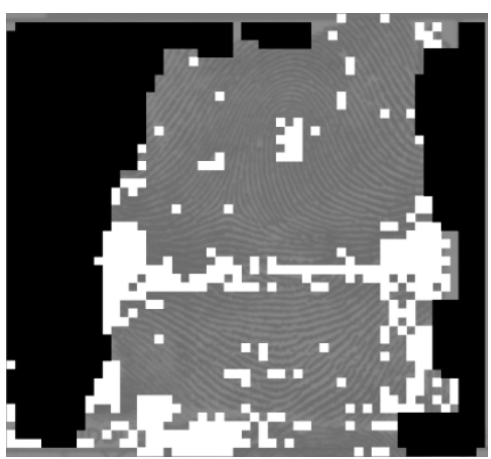

Figure 4. Noise areas of the foreground are identified and labelled The white coloured blocks indicate the noise areas 
(Note: the input image is referred to the extracted foreground image in Figure 1(a) above)

After the background, foreground, and noise regions are detected, further noise regions are enhancend using methods adopted from [16].

\section{EXPERIMENT RESULT AND DISCUSSION}

As mentioned in the previous chapter, fingerprint is segmented using a combination of local mean value and local variance of orientation fields' gradient magnitude. In order to measure the performance of the proposed segmentation technique in terms of visual inspection; five different fingerprint qualities viz. good, dry, wet, low contrast, and stain are used. This similar criterion was also used in [11]. Figure 5 to Figure 9 show five sets of fingerprints taken before and after the segmentation process that represent the above situation.
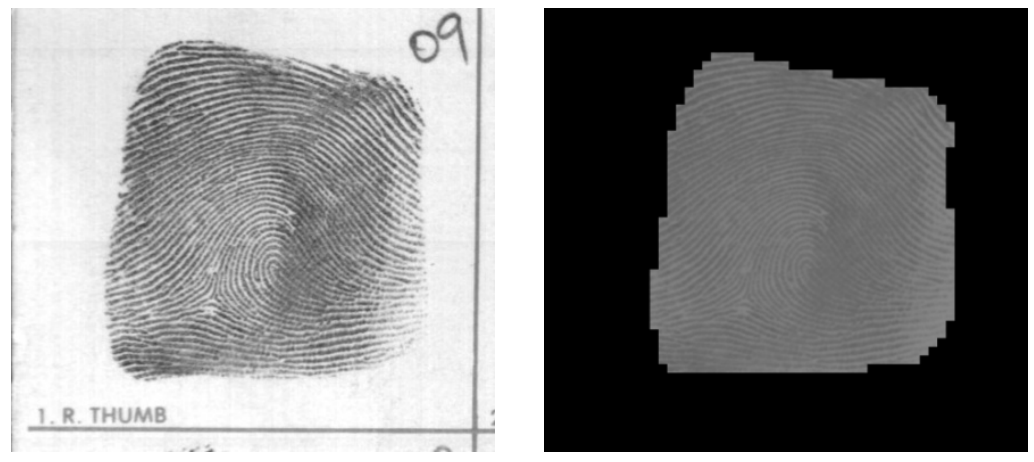

Figure 5. Result of the segmentation process of good quality fingerprint
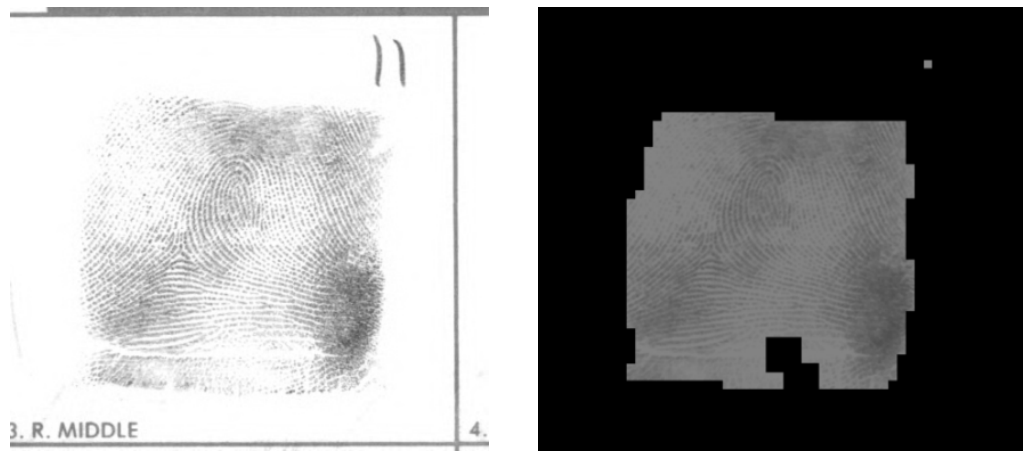

Figure 6. Result of the segmentation process of dry fingerprint
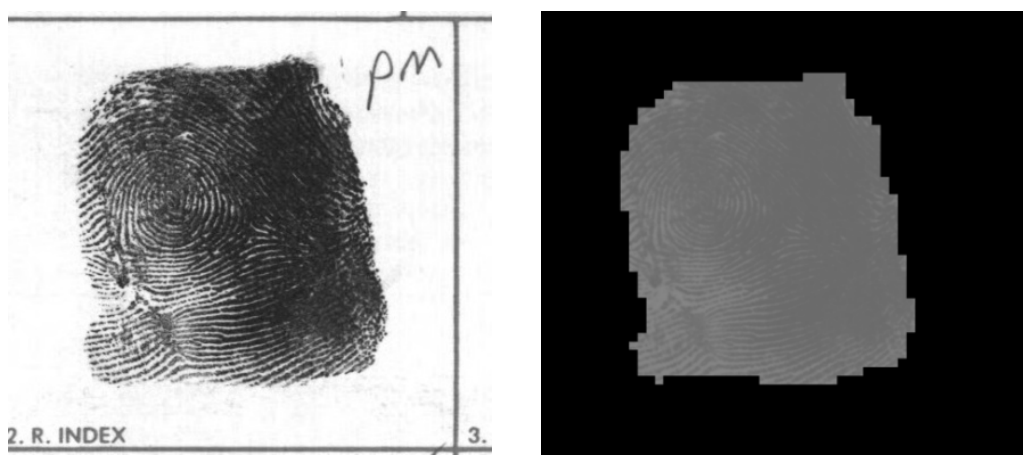

Figure 7. Result of the segmentation process of wet fingerprint 

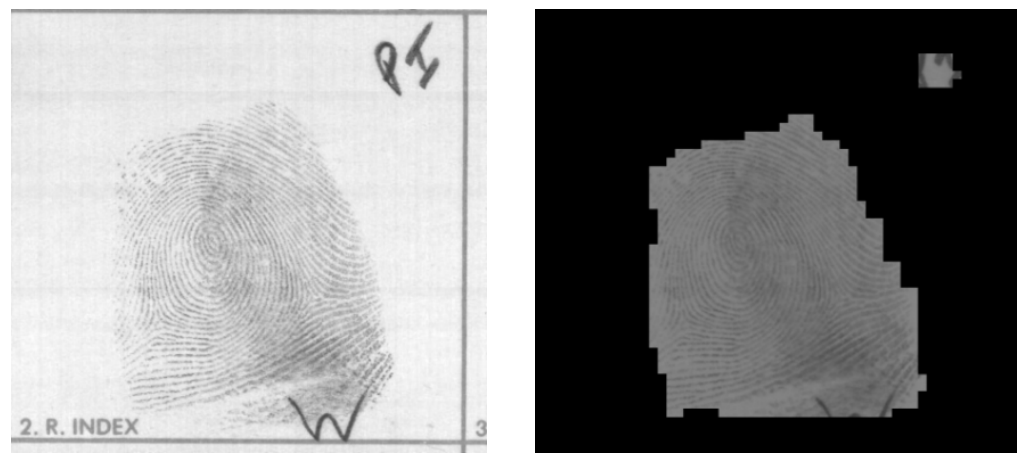

Figure 8. Result of the segmentation process of low contrast fingerprint
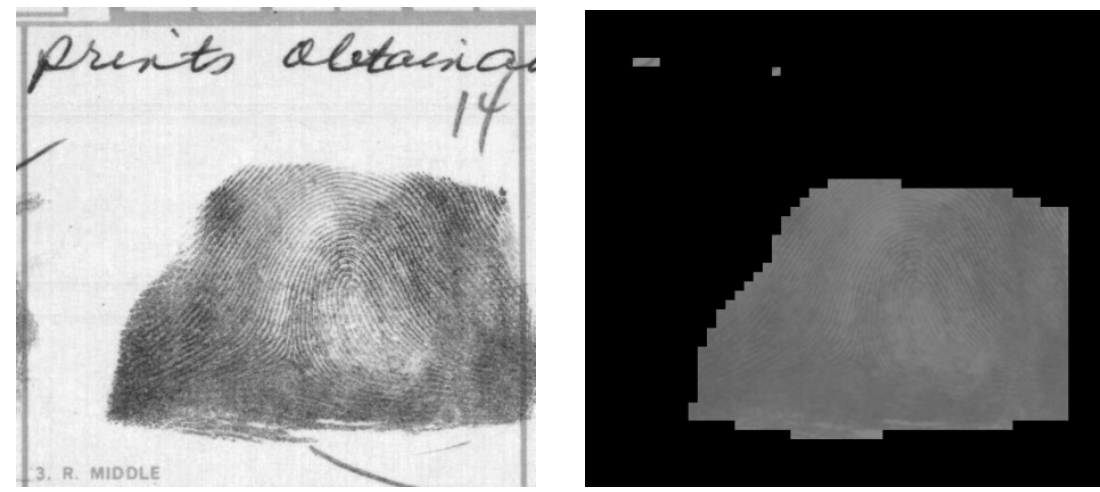

Figure 9. Result of the segmentation process of fingerprint which contain ink stains and handwritten characters

Overall, based on the above figures, the proposed segmentation technique has performed exceptionally well in most cases especially for good, dry and wet prints. The foregrounds are well separated from the background. However, in some low contrast prints, there are certain areas of the foregrounds have been wrongly marked as backgrounds. Likewise, for stain prints, there are some areas of background have been falsely labelled as foreground. Despite the imperfections, the segmented images or foregrounds are definitely well suited to facilitate the subsequence post-processing including ridge orientation field estimation and singular point detection.

Beside the human inspection, which is considered as a qualitative measure, alternatively, the assessment can be carried out quantitatively such as by counting the number of false and missed fingerprint features like minutiae or singular points [2]. As for the singular points; the performance is measured according to the ratio of number of true singular points that have been discarded to the total number of genuine singular points that existed in the print. In other words, this measurement is equivalent of the percentage of the discarded genuine singular points. Alternatively, the assessment can also be done according to the ratio between number of falsely accepted singular points and total number of genuine singular points.

Thus, in order to evaluate the performance of the proposed segmentation technique in terms of the above quantitative measure, an experiment is set up using 500 prints of the NIST-DB14 (i.e. f0000001 to f0000500). In addition to that, the technique is also benchmarked against several well established segmentation methods including local mean of grey-scale based technique, local variance of gradient magnitude, and a combination of local mean of gradient magnitude and block coherence approach by Zhang and Yan. The corresponding results are given in Table 1. 
Table 1. Performance results of various fingerprint segmentation methods from singular points detections points of view

\begin{tabular}{lcccc}
\multicolumn{1}{c}{ Method } & MC (\%) & MD (\%) & FC (\%) & FD (\%) \\
\hline $\begin{array}{l}\text { Local mean of greyscales based. } \\
\text { Combination local mean of gradient magnitude and block } \\
\text { coherence. }\end{array}$ & 16.39 & 36.99 & 1.05 & 0.75 \\
$\begin{array}{l}\text { Local variance of gradient magnitude. } \\
\text { Combination of local mean and local variance of gradient }\end{array}$ & 4.51 & 29.32 & 2.86 & 0.90 \\
magnitude (the proposed method). & & 14.44 & 11.73 & \multirow{2}{*}{5.21} \\
\hline MC : Miss Rate of Cores (i.e. discarded true cores). & & & \\
MD : Miss Rate of Deltas (i.e. discarded true deltas). & & & \\
FC : False alarm rate of Cores (i.e. falsely accepted cores). & & & \\
FD : False alarm rate of Deltas (i.e. falsely accepted deltas). & &
\end{tabular}

Table 1 shows that the local mean of greyscale based method has performed badly in terms of Miss Rate percentages of both core and delta. This was due to the fact that all the bright areas of the foreground are assigned to the background by blackening the areas. Ironically, it has however resulted in good performance in terms of False alarm rates of both core and delta. In fact, these FC and FD are actually artificial and unreliable because quite sizeable area of foreground was blackened and thus became background, hence is no longer considered as a search area when seeking for cores and deltas. As a result some cores and deltas either genuine or fake are vanished (see Figure 10(a) - (a2)).

Similarly, the same situation applied to Zang and Yan, but this time noise patches are now considered as rejected areas and therefore become off limit when searching for the existence of possible singular points. This is a self-explained reaction on why both the FC and FD attained were impressive. This move however is considered risky because cores and deltas may exist in the areas. Therefore, like the local mean method, the FC and FD are disputable. Figure 11(b)-(b2) depicts the scenario.

As for the local variance of gradient magnitude method, it has achieved $4.51 \%$ and $14.44 \%$ for MC and MD respectively, which are considered better than that of both local mean and Zang and Yan methods. However, its FC and FD are worsening to $11.73 \%$ and $4.21 \%$, respectively. This is attributed to the noise patches of the foreground, which are now counted in the segmentation process, as opposed to the above Zang and Yan method. Figure 12(c)-(c2) demonstrates the above results.

Finally, as for the proposed method whereby mean of greyscale and variance of gradient magnitude are used hand-in-hand to accommodate problematic areas including noise, dark grey, and bright patches in order not to unintentionally leave out any singular points because they may exist in those areas. Here, the key idea is that; an area is blackened if and only if both mean and variance have blackened the area. Consequently, the area now becomes background, and thus is no longer considered in the segmentation process. The above results have demonstrated that MC, FC and FD are markedly improved with $4.81 \%$, $5.86 \%$ and $2.11 \%$, respectively. However, MD is worsened, and this is due to the fact that most of the deltas are located near the edges of the fingerprints (Figure 13(d)-(d1)). Nevertheless, this weakness can be rectified by using structure shape of the orientation fields.

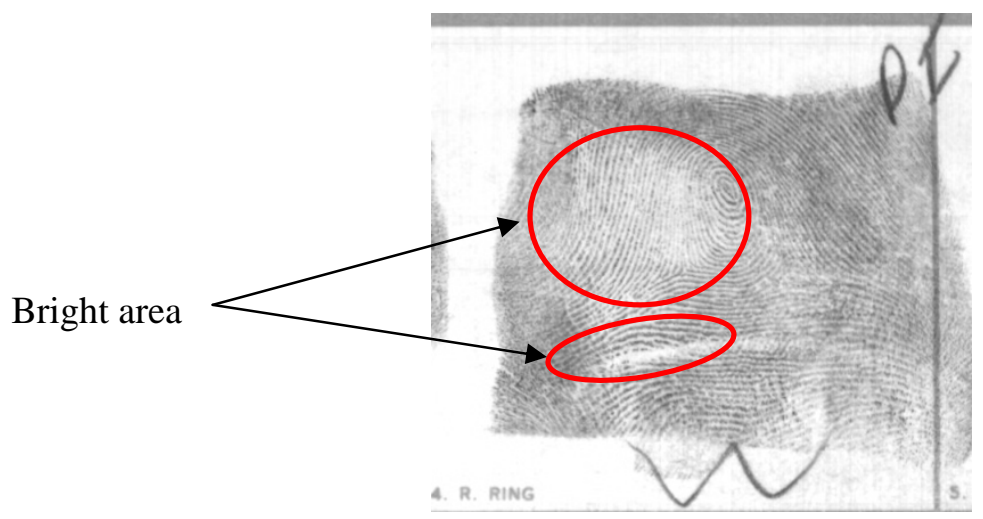

(a) 


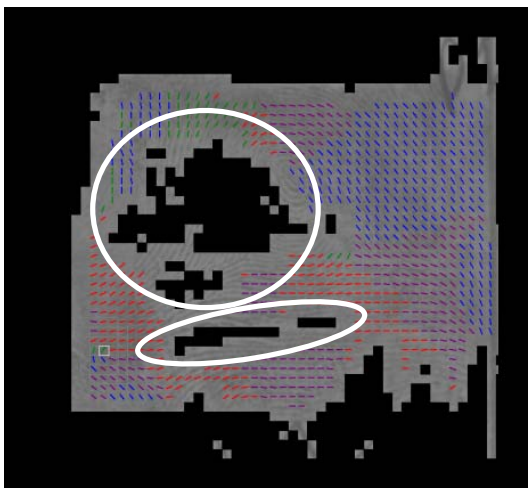

(a1)

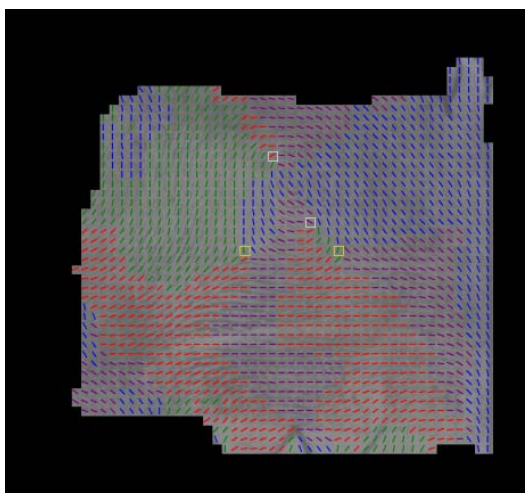

(a1)

Figure 10. On segmentation results: Local Mean Versus the proposed method. (a) Original fingerprint; (a1) outcome of local mean method; (a2) outcome of proposed method.

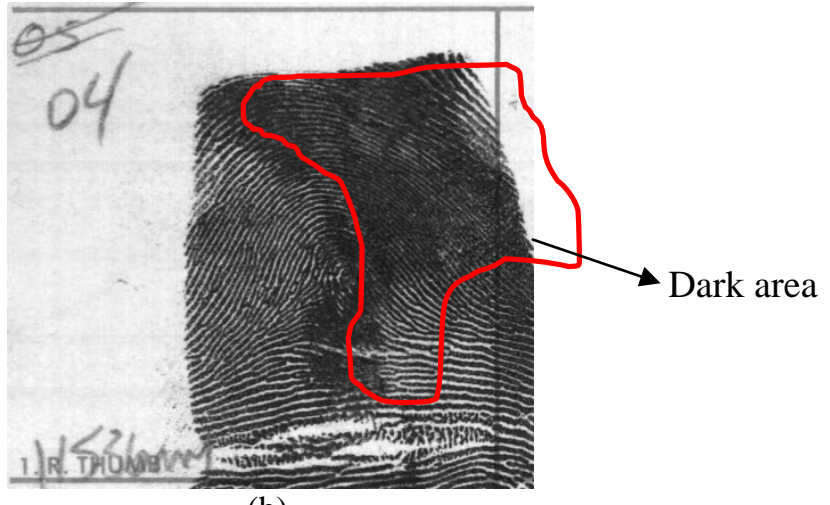

(b)

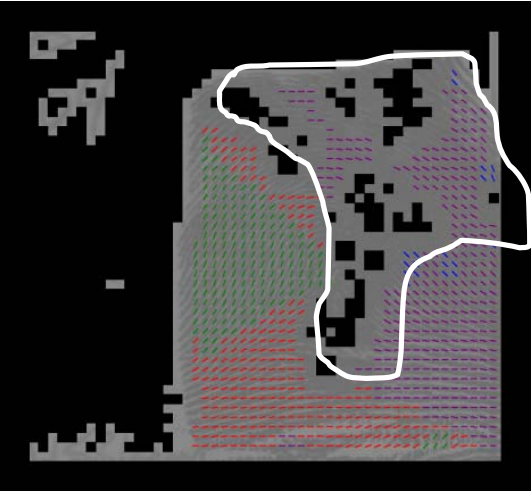

(b1)

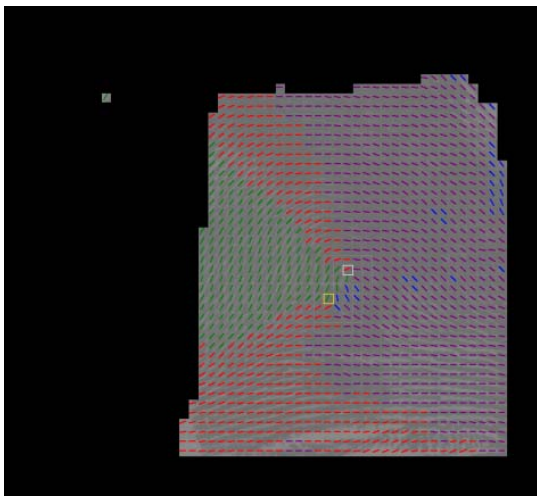

(b2)

Figure 11. On segmentation results: Zang and Yan Versus the proposed method. (b) Original fingerprint; (b1) outcome of Zang and Yan method; (b2) outcome of proposed method 


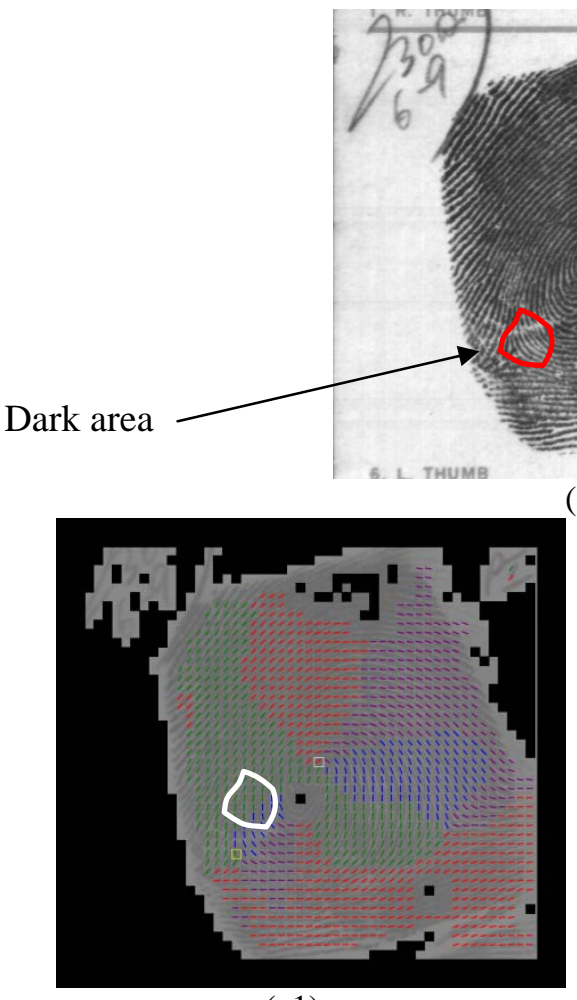

(c1) (c)

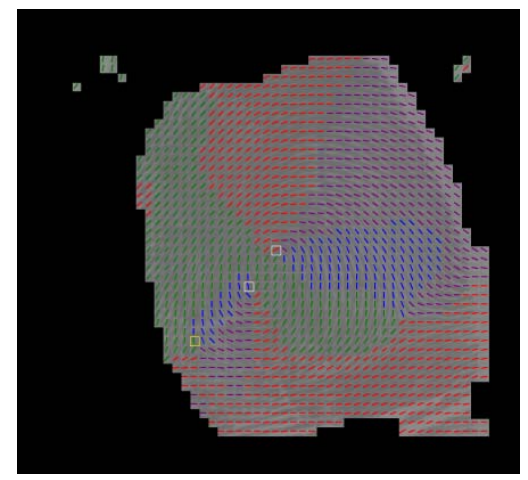

(c2)

Figure 12. On segmentation results: local variance of gradient magnitude method Vs the proposed method. (c) Original fingerprint; (c1) outcome of local variance of gradient magnitude method; (c2) outcome of the proposed method

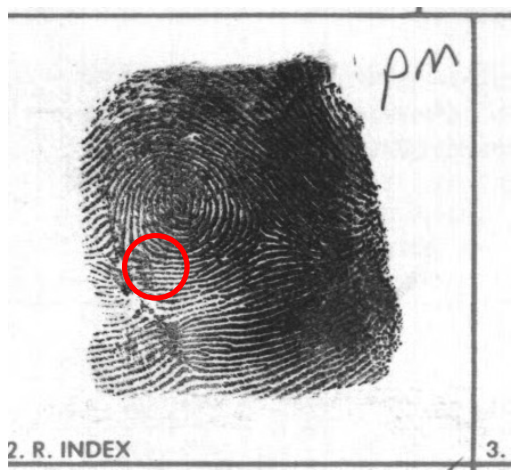

(d)

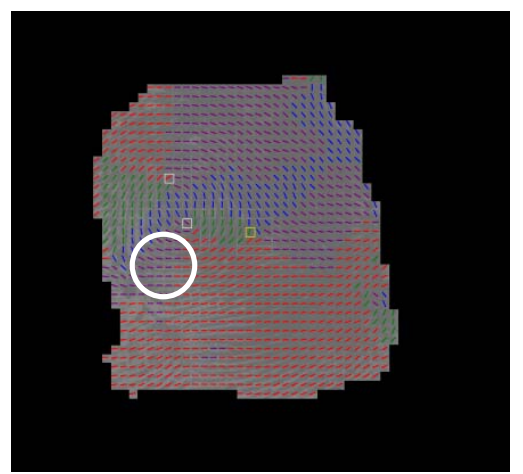

(d1)

Figure 13. On segmentation results: outcome of the proposed segmentation method (d) Original fingerprint; (d1) outcome of the proposed method

\section{CONCLUSION}

On the base of gradient magnitude and coherence features, the new approach to segment fingerprint image is proposed. This technique has successfully extracted the foreground from its background and marked the noise area in foreground of the fingerprint image. Experiments show that our method is improved compared to several methods, i.e. local mean of grey scales method, combination local mean of gradient magnitude and block coherence, and local variance of gradient magnitude method. Overall, the proposed segmentation technique has performed exceptionally well in most cases especially for good, dry and wet prints. 


\section{REFERENCES}

[1] Bazen A.M. and Gerez S.H. (2000). Directional Field Computation for Fingerprint Based on the Principal Component Analysis of Local Gradients. ProRISC 2000 Workshop on Circuits, Systems and Signal Processing, Veldhoven, the Netherlands.

[2] Bazen A.M. and Gerez S.H. (2001). Segmentation of Fingerprint Images. ProRISC 2001 Workshop on Circuits, Systems and Signal Processing, Veldhoven, the Netherlands.

[3] Candela G.T., Grother P.J., Watson C.I., Wilkinson R.A., and Wilson C.L. (1995). PCASYS-A Pattern-level Classification Automation System for Fingerprints. NIST Technical Report NISTIR 5647.

[4] Cappelli R. and Maltoni D. (2009). On the Spatial Distribution of Fingerprint Singularities. IEEE Transactions on Pattern Analysis and Machine Intelligence, vol. 31, no. 4.

[5] Chen X., Tian J., Cheng J., and Yang X. (2004). Segmentation of Fingerprint Images using Linear Classifier. EURASIP Journal on Applied Signal Processing, vol. 2004, no. 4, pp. 480-494.

[6] Feng W., Xiuyou W., and Lin X. (2009). An Improved Fingerprint Segmentation Algorithm based on Mean and Variance. Intelligent System and Applications (ISA). IEEE International Workshop, 23-24 May 2009.

[7] Hong L., Wan Y., and Jain A.K. (1998). Fingerprint Image Enhancement: Algorithm and Performance Evaluation. IEEE Transactions on Pattern Analysis and Machine Intelligence, vol. 20, no. 8, pp. 777-789.

[8] Klein S., Bazen A., and Veldhuis R. (2002). Fingerprint Image Segmentation based on Hidden Markov Models. Proceedings of 13th Annual Workshop on Circuits, Systems, and Signal Processing, vol. 2002, pp. 310-318.

[9] Ma J., Jing X., Zhang Y., Sun S., and Huang H. (2010). Simple Effective Fingerprint Segmentation Algorithm for low Quality Images. Proceeding of International conference-Broadband Network and Multimedia Technology (ICBNMT), $3^{\text {rd }}$ IEEE International Conference, pp. 855-859.

[10] Maio D. and Maltoni D. (1997). Direct Gray-Scale Minutiae Detection in Fingerprints. IEEE Transactions on Pattern Analysis and Machine Intelligence, vol. 19, no. 1.

[11] Maltoni D., Maio D., Jain A.K., and Prabhakar S. (2009). Handbook of Fingerprint Recognition. Second edition. Springer-Verlag London Limited.

[12] Mehtre B.M., Murthy N.N., Kapoor S., and Chatterjee B. (1987). Segmentation of Fingerprint Images Using the Directional Images. Pattern Recognition, vol. 20, no. 4, pp. 429-435.

[13] Mehtre B.M. and Chatterjee B. (1989). Segmentation of Fingerprint Images a Composite Method. Pattern Recognition, vol. 22, no. 4, pp. 381-385.

[14] Qi J. and Xie M. (2008). Segmentation of Fingerprint Images Using the Gradient Vector Field. Cybernetics and Intelligent System. IEEE Conference, pages: 543-545.

[15] Ratha N.K., Chen S., and Jain, A.K. (1995). Adaptive Flow Orientation Based Feature Extraction in Fingerprint Images, Pattern Recognition, vol. 28, no. 11, pp. 1657-1672.

[16] Saparudin. (2012). An Automic Fingerprint classification Technique Based on Singular Points and Sructure Shape of Orientation Fields. Thesis in Computer Science, Universiti Teknologi Malaysia: Johor Baru. 241.

[17] Teixeira R.F.S and Leite N.J. (2011). Unsupervised Fingerprint Segmentation Based on Multiscale Directional Information. CIARP 2011, LNCS 7042, pp. 38-46. Springer-Verlag Berlin Heidelberg.

[18] Wu C., Tulyakov S., and Govindaraju V. (2007). Robust Point-Based Feature Fingerprint Segmentation Algorithm. ICB 2007, LNCS 4642, pp. 1095-1103. Springer-Verlag Berlin Heidelberg.

[19] Yin J.P., Zhu E., Yang X.J., Zhang G.M., and Hu C.F. (2007). Two Steps for Fingerprint Segmentation. Journal Image and Vision Computing, vol. 25, no. 9, pp. 1391-1403.

[20] Yu C., Xie M., and Qi J. (2008). An Effective Algorithm for Low Quality Fingerprint Segmentation. IEEE Proc. of $3^{\text {rd }}$ Int. Conf. on Intelligent System and Knowledge Engineering.

[21] Zhang Q. and Yan H. (2007). Fingerprint Orientation Field Interpolation based on The Constrained Delaunay Triangulation. International Journal of Information and Systems Sciences, vol. 3, no 3, pp. 438-452.

[22] Zhu E., Yin J., Hu C., and Zhang G. (2006). A Systematic Method for Fingerprint Ridge Orientation Estimation and Image Segmentation. Pattern Recognition, vol. 39, pp. 1452-1472.

[23] Zhu E., Zhang J., Yin J., Zhang G., and Hu C. (2006a). Removing the Remaining Ridges in Fingerprint Segmentation. Journal of Zhejiang University Science A, 2006 7(6), pp. 976-983.

[24] Kass M. and Witkin A. (1987). Analyzing Oriented Patterns. Computer Vision, Graph and Image Processing, vol. 37, no. 3, pp. 362-385. 